EPJ Web of Conferences 47, 06007 (2013)

DOI: $10.1051 /$ epjconf/20134706007

(C) Owned by the authors, published by EDP Sciences, 2013

\title{
Purple dwarfs: New L subdwarfs from UKIDSS and SDSS
}

\author{
Z.H. Zhang ${ }^{1, a}$, D.J. Pinfield ${ }^{1}$, B. Burningham ${ }^{1}$, H.R.A. Jones ${ }^{1}$, \\ A.C. Day-Jones ${ }^{2}$, F. Marocco ${ }^{1}$, J. Gomes ${ }^{1}$ and M.C. Galvez-Ortiz ${ }^{3}$ \\ ${ }^{1}$ Centre for Astrophysics Research, University of Hertfordshire, Hatfield AL10 9AB, UK \\ ${ }^{2}$ Department of Astronomy, Universidad de Chile, Casilla postal 36D, Santiago, Chile \\ ${ }^{3}$ Centro de Astrobiologia - INTA-CSIC
}

\begin{abstract}
The first L subdwarf was a discovered only ten years ago. Less than ten L subdwarfs been published in the literature to date. Metal-poor ultracool atmospheres has not been well understood. Halo mass function cross substellar limit has not been measured. We used UKIDSS and SDSS to search for L subdwarfs. We have confirmed some new L subdwarfs and are following up more candidates with ground based large telescopes. We discussed spectral features of L subdwarfs and halo brown dwarfs.
\end{abstract}

\section{INTRODUCTION}

L subdwarfs are a mixture of lowest mass stars and brown dwarfs with subsolar abundance and $T_{\text {eff }}$ $\leq 2500 \mathrm{~K}$. L subdwarfs are kinematically associated with Galactic halo and thick disk and form the low mass end of the stellar Population II. They have higher proper motion and tangential velocity than L dwarfs. L subdwarfs exhibit characteristic spectral signatures of strong metal hydrides $(\mathrm{CaH}, \mathrm{MgH}$, $\mathrm{AlH}$ and $\mathrm{FeH}$ ), weak or absent metal oxides (TiO, $\mathrm{VO}$ and $\mathrm{CO}$ ), and enhanced collision-induced $\mathrm{H}_{2}$ absorption $\left(\mathrm{CIA} \mathrm{H}_{2}\right.$ ). Near infrared flux of $\mathrm{L}$ subdwarfs is suppressed due to the CIA $\mathrm{H}_{2}$. $\mathrm{L}$ subdwarfs are red in optical, and bluer than same effective temperature L dwarfs in near infrared, thus could also be called as purple dwarfs.

$\mathrm{L}$ subdwarfs are very rare compare with $\mathrm{L}$ dwarfs. There are over one thousand $\mathrm{L}$ dwarfs been discovered while there only seven L subdwarfs been published [1-6]. Only 2MASS J05325346+8246465 (J0532) has estimated mass (based on models of [7, 8]) just blow the sustained hydrogen burning limit, and is therefore a possible substellar subdwarf or a halo brown dwarf. The low metallicity ultracool atmosphere models (e.g. [9]) have not been tested properly, the L subdwarf classification scheme has not been established due to the lack of known L subdwarfs. Currently L subdwarfs are classified by compare their optical spectra with that of L dwarfs. Model spectra show both low metallicity and high gravity suppress the near infrared flux of L subdwarfs, and shift the flux peak to shorter wavelength [9]. Near infrared spectra must be considered in order to characterize L subdwarfs properly.

It is critical to discover a sample of $\mathrm{L}$ subdwarfs with different temperature and metallicity. L subdwarfs could not only help us to understand metal-poor ultracool atmospheres but also the formation and structure of the Galactic halo through study of the luminosity and mass function and kinematics. We started a project to identify a sample of L subdwarfs with UKIDSS and SDSS. We have

\footnotetext{
ae-mail: zenghuazhang@gmail.com
}

This is an Open Access article distributed under the terms of the Creative Commons Attribution License 2.0, which permits unrestricted use, distribution, and reproduction in any medium, provided the original work is properly cited. 

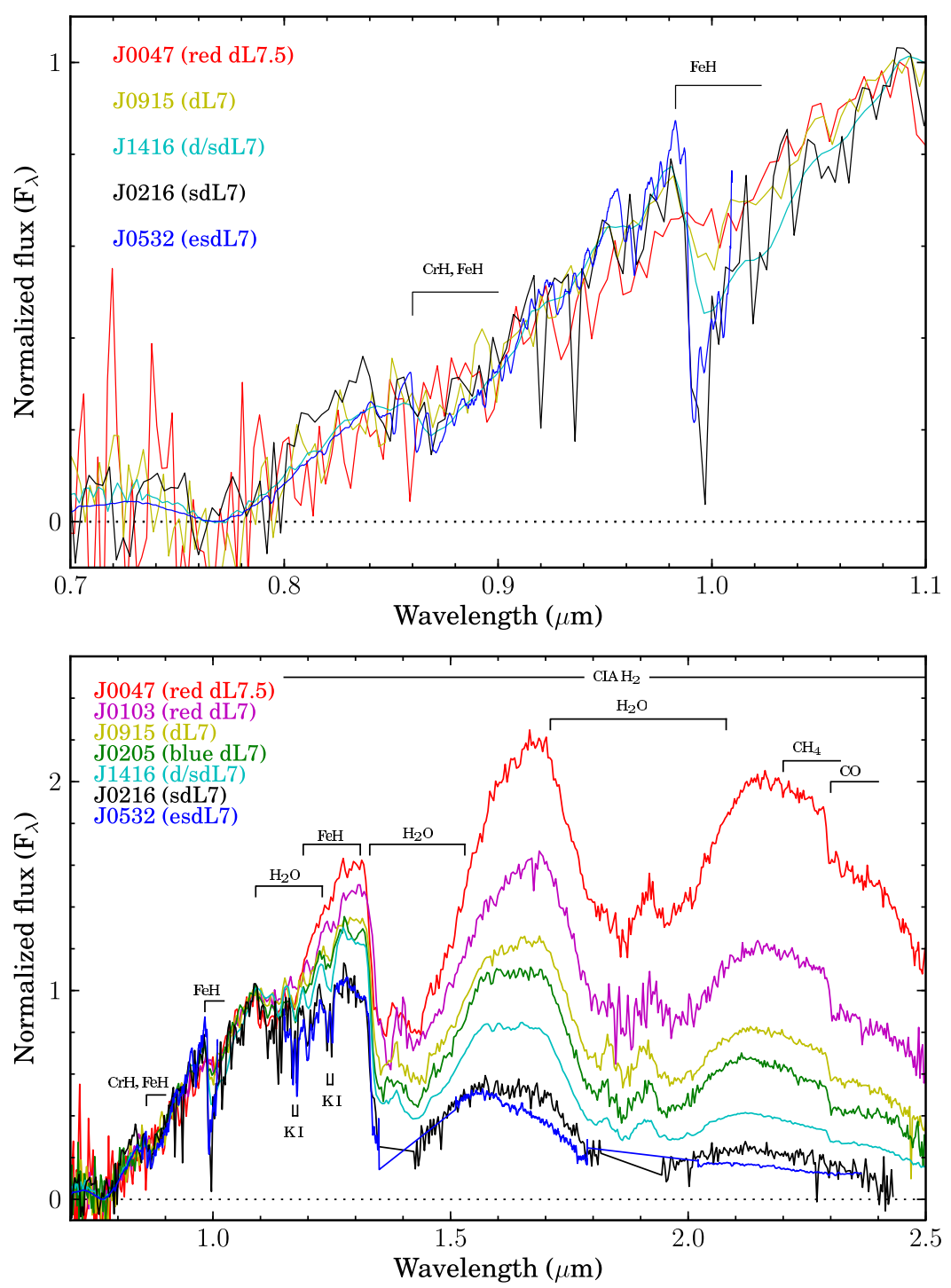

Figure 1. Metallicity sequences of optical and near infrared spectra of L7 dwarfs and subdwarfs. The absence $2.3 \mu \mathrm{m} \mathrm{CO}$ band which presents in spectra of M7 - T1 dwarfs might be a indicator of L subdwarfs. Spectra are normalized at $1.08 \mu \mathrm{m}$.

confirmed three new L subdwarfs and followed up 10 candidates with the Magellan Telescope and Gran Telescopio Canarias. We are also following up another 41 candidates with the Gran Telescopio Canarias.

\section{METAL CLASSES OF L SUBDWARFS}

The overall profile of optical spectra of L subdwarfs is less affected by the chemical abundance because the dust cloud delays the suppressing of spectra below one micron [9]. Thus optical spectra could represent the effective temperature better than near infrared spectra. The infrared spectral of L subdwarfs/dwarfs are sensitive to both chemical abundance and effective temperature. Figure 1 top shows optical spectra of L7 dwarfs/subdwarfs. Similar overall profile indicates similar effective 


\section{Hot Planets and Cool Stars}
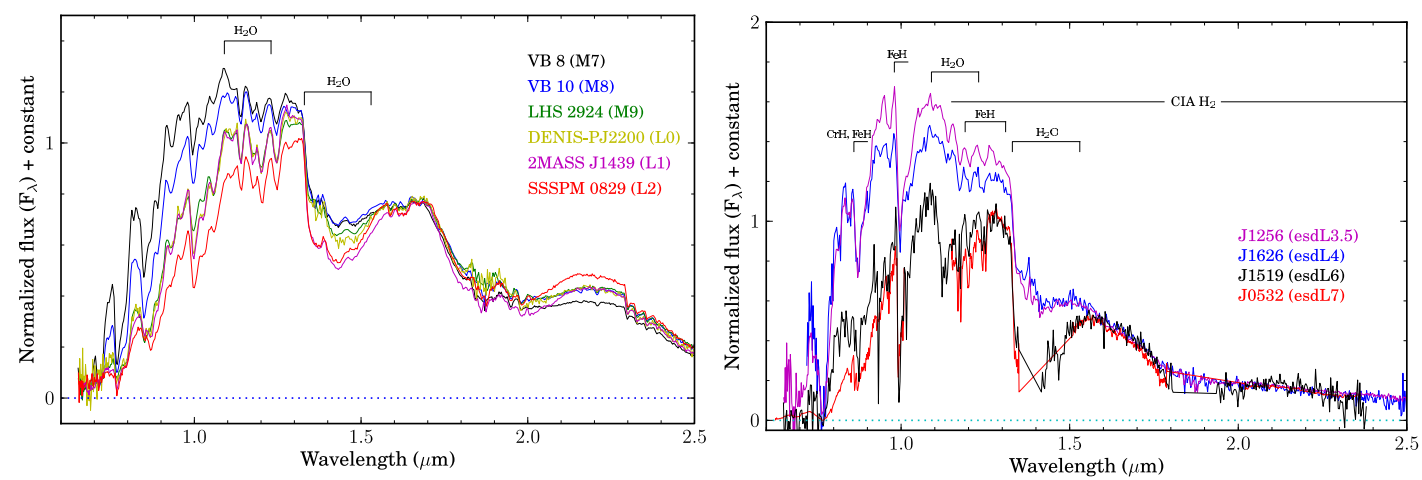

Figure 2. Left: spectra of M7 - L2 dwarfs. Right: optical and near infrared spectra of four L type extreme subdwarfs. Deep $1.4 \mu \mathrm{m} \mathrm{H}_{2} \mathrm{O}$ absorption in ULAS 1519 and 2MASS J0532 which are different from 2MASS J1626 indicates that they might be halo brown dwarfs or substellar subdwarfs. All spectra are normalized at $1.6 \mu \mathrm{m}$.

temperature, while stronger metal hydrides (e.g. FeH) indicates lower metallicity. Figure 1 bottom shows the near infrared spectra of L7 dwarfs/subdwarfs. A smooth suppressing of $J, H$ and $K$ band spectra moving across objects classified as, red L7, normal L7, blue L7, d/sdL7, sdL7 and esdL7. This spectral sequence also represents a sequence of brown dwarfs with similar effective temperature but different masses (from the lowest to the highest mass brown dwarfs). This is an intuitive proof of the evolution of brown dwarfs.

The most notable features of an L7 subdwarf spectrum that differ from an L7 dwarf spectrum are the much stronger $\mathrm{FeH}$ lines and absence of the $\mathrm{CO}$ band around $2.3 \mu \mathrm{m}$. The $2.3 \mu \mathrm{m} \mathrm{CO}$ band appears in spectra of M7 to T1 dwarfs, and disappear after T2 due to the strengthening of $\mathrm{CH}_{4}$ absorption (Fig. 2 of [10]). The absence of $2.3 \mu \mathrm{m} \mathrm{CO}$ band in the spectra of known L subdwarfs suggests that it is an indicator of a subsolar abundance population.

\section{HALO BROWN DWARFS}

The most massive brown dwarfs have spectral types of late $\mathrm{M}$ and are usually found in $\sim 100 \mathrm{Myr}$ old young open clusters. Brown dwarfs in the Galactic disk are usually a few Gyr old start with spectral type of L0. After about 10 Gyr of cooling, brown dwarfs would have temperature lower than $\sim 1600 \mathrm{~K}$ (e.g. [8]). Thus spectral types of old brown dwarfs in the halo should start with late L types.

The esdL7 type J0532 is the first L subdwarf discovered and is the only one with theoretical predicated mass $\left(\sim 0.08 M_{\odot}\right)$ just below the hydrogen burning minimum mass. Figure 2 (left) shows spectra of M7 to L2 dwarfs which cover the star-brown dwarf transit region. $\mathrm{H}_{2} \mathrm{O}$ absorption bands are strengthening from M7 to L2. Figure 2 (right) shows spectra of four L subdwarfs. The depth of the $\mathrm{H}_{2} \mathrm{O}$ absorption band around $1.4 \mu \mathrm{m}$ in the spectrum of J1626 is similar to that seen in late M dwarfs, a few Gyr old lowest mass stars. While the $\mathrm{H}_{2} \mathrm{O}$ absorption depth (around $1.4 \mu \mathrm{m}$ ) of the spectrum of $\mathrm{J} 1519$ (Zhang et al. in preparation) is similar to that of early L dwarfs, a few Gyr old high mass brown dwarfs. The $1.15 \mu \mathrm{m} \mathrm{H}_{2} \mathrm{O}$ band in $\mathrm{J} 1519$ is also much stronger than that of J1626. With only two subtype difference, spectra of J1626 and J1519 are showing significantly different features. Could the strengthen of $1.4 \mu \mathrm{m} \mathrm{H}_{2} \mathrm{O}$ absorption band in $\mathrm{J} 1519$ be an indicator of a substellar subdwarf? If this is true J0532, J0616 [3], J1519 and J0216 (Zhang et al. in preparation) would be the first four brown dwarfs discovered in the Galactic halo. Further analysis based on the latest model grids and new astrometric observations are needed to confirmed this hypothesis. 
EPJ Web of Conferences

\section{References}

[1] Burgasser A. J., et al., ApJ, 592, 1186 (2003)

[2] Burgasser A. J., ApJ, L73, 614 (2004)

[3] Cushing M. C., Looper D., Burgasser A. J., Kirkpatrick J. D., Faherty J., Cruz K. L., Sweet A., Sanderson R. E., ApJ, 696, 986 (2009)

[4] Sivarani T., Lépine S., Kembhavi A. K., Gupchup J., ApJ, 694, L140 (2009)

[5] Lodieu N., Zapatero Osorio M. R., Martín E. L., Solano E., Aberasturi M., ApJ, 708, L107 (2010)

[6] Lodieu N., Espinoza Contreras M., Zapatero Osorio M. R., Solano E., Aberasturi M., Martín E. L., A\&A, 542, A105 (2012)

[7] Baraffe I., Chabrier G., Allard F., Hauschildt P. H., A\&A, 327, 1054 (1997)

[8] Burrows A., Hubbard W. B., Lunine J. I., Liebert J., RvMP, 73, 719 (2001)

[9] Witte S., Helling C., Hauschildt P. H., A\&A, 506, 1367 (2009)

[10] Burgasser A. J., ApJ, 659, 655 (2007) 